\title{
Intensive $v s$ conventional blood glucose control in critically ill patients
}

\author{
Sean M. Bagshaw, MD - Eric A. J. Hoste, MD, PhD • \\ Michael J. Jacka, MD
}

Received: 15 September 2009/ Accepted: 6 November 2009/Published online: 31 December 2009

(C) Canadian Anesthesiologists' Society 2009

\section{Article appraised}

Finfer S, Chittock DR, Su SY, et al. Intensive versus conventional glucose control in critically ill patients. The NICE-SUGAR Study Investigators. N Engl J Med 2009; 360: $1283-97 .^{1}$

\section{Critical care issue}

Hyperglycemia is common during critical illness and has been identified as a modifiable risk factor for increased morbidity and mortality in critically ill patients. Data from selected clinical trials suggest that intensive insulin therapy (IIT) and tight glycemic control (TGC) achieve a clinical benefit when targeting blood glucose (BG) in the range of 4.4$6.1 \mathrm{mmol} \cdot \mathrm{L}^{-1}$. However, reports of recently conducted randomized trials have shown conflicting results. Consequently, the NICE-SUGAR (Normoglycaemia in Intensive Care Evaluation and Survival Using Glucose Algorithm Regulation) trial was designed as a pragmatic multi-national multi-centre randomized "effectiveness" trial to evaluate the impact of IIT to achieve TGC on 90-day all cause mortality and several secondary morbidity outcomes. The NICESUGAR trial aimed to resolve existing concerns about the applicability of IIT to achieve TGC in critical illness. This article considers the NICE-SUGAR trial and discusses its key findings in the context of the published literature.

S. M. Bagshaw, MD $(\bowtie) \cdot$ M. J. Jacka, MD

University of Alberta, Edmonton, Canada

e-mail: bagshaw@ualberta.ca

E. A. J. Hoste, $\mathrm{MD}, \mathrm{PhD}$

Ghent University, Ghent, Belgium
As the optimum target range for blood glucose (BG) in critically ill patients remains unclear, the objective of this study was to evaluate whether intensive glucose control in critical illness is associated with improved clinical outcomes. The design was a multi-national multi-centre randomized controlled trial. The setting involved 42 participating intensive care units (ICUs) across Australia, New Zealand, Canada, and the United States. Critically ill adult subjects met the eligibility requirements if, within $24 \mathrm{hr}$ after admission to an ICU, they were expected to require treatment in the ICU for three or more consecutive days. Subjects were randomized to receive either intensive glucose control with a target blood glucose range of $4.5-6.0 \mathrm{mmol} \cdot \mathrm{L}^{-1}$ or conventional glucose control with a target blood glucose range of $10.0 \mathrm{mmol} \cdot \mathrm{L}^{-1}$ or less. The primary endpoint was allcause mortality 90 days after randomization. Of the 6,104 subjects who were randomized, 3,054 patients were randomized to undergo intensive glucose control and 3,050 patients received conventional glucose management. Data with regard to the primary outcome at day 90 were available for over $98 \%$ of the subjects evaluated. The two groups had similar characteristics at baseline. The authors reported that 829 patients $(27.5 \%)$ in the intensive-control group and 751 (24.9\%) patients in the conventional-control group died (odds ratio [OR] for intensive control, 1.14; 95\% confidence intervals $[\mathrm{CI}], 1.02-1.28 ; P=0.02)$. The treatment effect was similar when comparing operative (surgical) patients and non-operative (medical) patients (odds ratio for death in the intensive-control group, 1.31 and 1.07 , respectively; $P=0.10)$. Severe hypoglycemia, defined as a blood glucose level $<2.2 \mathrm{mmol} \cdot \mathrm{L}^{-1}$ was reported in $206(6.8 \%)$ of patients in the intensive-control group and $15(0.5 \%)$ of patients in the conventional-control group $(P<0.001)$. The groups were found to be similar regarding the median 
number of days in the ICU, hospital length of stay, and the median number of days of mechanical ventilation and renalreplacement therapy $(P=0.39)$. The authors concluded that intensive glucose control was associated with increased mortality amongst adults in the ICU. A blood glucose target of $10 \mathrm{mmol} \cdot \mathrm{L}^{-1}$ or less was associated with a lower mortality than a target glucose concentration of 4.5$6.0 \mathrm{mmol} \cdot \mathrm{L}^{-1}$.

\section{Commentary}

Elevated blood glucose (BG) levels and stress hyperglycemia have been identified as modifiable risk factors for adverse outcomes in critically ill patients. ${ }^{2}$ Randomized trials of intensive monitoring and insulin therapy (IIT) in critically ill patients have shown improvements in morbidity and mortality with tight glycemic control (TGC)..$^{3-6}$ For TGC to exert its clinical benefit, data from selected trials suggest that $\mathrm{BG}$ values must be maintained in the range of $4.4-6.1 \mathrm{mmol} \cdot \mathrm{L}^{-1} \cdot 3,4$ Based on this evidence, use of IIT to achieve TGC has been advocated to improve outcomes for critically ill patients. ${ }^{7,8}$

There have, however, been concerns regarding the broad application of IIT and TGC in critical illness. ${ }^{9-12}$ Specifically, concerns have been raised about the methodology and overall generalizability of the two highest profile IIT trials reported by Van den Berghe et al. ${ }^{3,4}$ In particular, these trials were both single centre open-label trials where $87 \%$ of patients received parenteral nutrition. The observed mortality was high, specifically for a cohort predominantly characterized by elective cardiac surgery, ${ }^{4}$ and the estimate of treatment effect on in-hospital death was improbably large (e.g., relative risk reduction $\sim 32 \%$ ). Moreover, the second IIT trial performed in a medical ICU population was negative, and a survival benefit was demonstrated only following a planned subgroup analysis (duration of stay $>72 \mathrm{hr}$ ). ${ }^{3}$

Importantly, concern had also arisen that TGC with IIT is associated with unacceptably high rates of hypoglycemia. ${ }^{13}$ In the two IIT trials by Van den Berghe et al., hypoglycemia ( $\mathrm{BG}<2.2 \mathrm{mmol} \cdot \mathrm{L}^{-1}$ ) occurred in 5.1 and $18.7 \%$ of patients, respectively. ${ }^{3,4}$ Recently, the VISEP (Volume Substitution and Insulin Therapy in Severe Sepsis) trial, a multi-centre randomized trial comparing IIT with conventional therapy in critically ill septic patients, was terminated early due to lack of evidence of survival benefit and greater hypoglycemia in those allocated to IIT (17.0\% vs $4.1 \%$; $P<0.001) .{ }^{14}$ Several additional trials conducted across a range of critically ill populations have also failed to show a survival benefit and have shown an increased risk of hypoglycemia with IIT. ${ }^{13,15-18}$ The key concern from these observations is defining what constitutes the optimal and safest target for BG in critically ill patients to both improve clinical outcomes and prevent the adverse consequences of hypoglycemia. ${ }^{19}$

Finally and equally concerning, the evidence from the two IIT trials by Van den Berghe et al., representing the vast majority of patients subjected to intervention with IIT, had rapid and wide dissemination into clinical practice guidelines and incorporation as a benchmark quality indicator in critical care, leading to the development of numerous industry-funded applications (e.g., monitoring devices, specialized software).

\section{Study relevance}

The NICE-SUGAR (Normoglycaemia in Intensive Care Evaluation and Survival Using Glucose Algorithm Regulation) trial was designed as a pragmatic multi-national multi-centre randomized "effectiveness" trial to evaluate the impact of IIT to achieve TGC on morbidity and mortality in a large heterogeneous cohort of critically ill patients. ${ }^{1}$ Moreover, NICE-SUGAR was specifically aimed to validate the observations of the studies by Van den Berghe et al. and to definitively address the existing concerns on the broad use of IIT to achieve TGC in critical illness. ${ }^{19}$

The key finding from NICE-SUGAR was an observed increased risk of all-cause mortality at 90 days for critically ill patients allocated to intensive $v s$ conservative glucose control (27.5\% vs 24.9\%, OR 1.14; 95\% CI, 1.02-1.28; $P=0.02$ ). This finding was certainly at odds with prior trials that showed either reduced mortality ${ }^{3,4}$ or no impact on survival ${ }^{13,14,20}$ with IIT (of interest, in the second IIT trial by Van den Berghe et al., mortality was higher for those allocated to IIT with an ICU length of stay $<72 \mathrm{hr}$ ). ${ }^{3}$ This observed increased risk of death was also largely consistent (though not always statistically significant) across a priori planned subgroups, including primary surgical admissions and patients with diabetes mellitus, severe sepsis, and high illness severity (APACHE II score $\geq 25$ ). In contrast, there were non-significant trends toward benefit for IIT in critically ill trauma patients and those receiving corticosteroids. While these findings are of interest, they remain hypothesisgenerating only and would require further verification prior to formulating any recommendations.

There was no difference between treatment groups regarding secondary outcomes, including 28-day mortality ( $22.3 \%$ for IIT vs $20.8 \%$ for conventional; $P=0.17$ ), the need for and/or duration of mechanical ventilation, the need for and/or duration of renal-replacement therapy (RRT), new organ dysfunction, red blood cell transfusion, or positive blood cultures. However, those allocated to IIT had a 15-fold increased risk of severe hypoglycemia (BG $<2.2 \mathrm{mmol} \cdot \mathrm{L}^{-1}$ ) compared with conventional therapy $(6.8 \%[n=206]$ vs $0.5 \%[n=15]$, OR $14.7 ; 95 \%$ CI, 
9.0-25.9; $P<0.001)$. Notably, the observed rate of hypoglycemia associated with IIT was lower in NICESUGAR compared with prior studies, despite a similar relative risk. ${ }^{3,4,13,14,20}$

\section{Analysis of methodology}

The obvious strength of the NICE-SUGAR trial was its carefully designed, practical "effectiveness" parallel-group randomized clinical trial with transparent methodology, analysis, and reporting. The process for randomization was centralized and concealed; the analysis was performed by intention-to-treat, and attrition was low $(<1.5 \%)$. NICESUGAR also employed a standardized computer-based treatment algorithm accessible on centralized servers for use by all participating centres. Examination of baseline clinical characteristics shows that patient enrolment was appropriately balanced.

There are also limitations to NICE-SUGAR. The most important was an inability to blind study personnel to the treatment allocation. In addition, despite a standardized algorithm, a significant proportion of patients allocated to IIT had time-averaged BG levels above the target 4.5$6.0 \mathrm{mmol} \cdot \mathrm{L}^{-1}$. However, in the two trials by Van den Berghe, time-averaged BG levels were not reported; rather, these trials reported morning BG values as a surrogate for BG control. Accordingly, we are unable to directly compare time-averaged BG control in NICE-SUGAR with the trials by Van den Berghe et al. ${ }^{3,4}$ Likewise, when compared with the trials by Van den Berghe et al., the absolute difference in average BG levels between the IIT and control groups was smaller in NICE-SUGAR due to higher BG in the IIT group and lower target in the control group (8.1$10.0 \mathrm{mmol} \cdot \mathrm{L}^{-1}$ vs $10.0-11.1 \mathrm{mmol} \cdot \mathrm{L}^{-1}$ ). In addition, there were questions raised about the reliability and nonstandardized methods of BG measurements in NICESUGAR, in particular for the detection of hypoglycemia, due to the use of both blood gas analyzers and different point-of-care devices. ${ }^{21}$ The study was not designed to enable investigation of the biologic mechanisms that may account for the observed harm associated with IIT. Accordingly, the NICE-SUGAR study investigators are appropriately cautious about the plausible factors to explain the observed increased mortality, including studyspecific methodological factors, reduced BG levels, increased administration of insulin, or the differential occurrence of hypoglycemia.

\section{External validity}

The NICE-SUGAR study evaluated IIT in a heterogeneous population of critically ill patients. The inclusion criteria were broad (i.e., any ICU admission with an expected duration of stay $>72 \mathrm{hr}$ and an arterial line), and there were relatively few criteria for exclusion. The study was conducted across three countries and in 42 centres. The trial was large and enrolled a sufficient number of patients to give it greater statistical power to detect clinically important outcomes than all prior trials combined. In addition, patients enrolled in NICE-SUGAR were predominantly fed by the enteric route (approximately $70 \%$ ), a practice more consistent with current evidence-based guidelines. These attributes imply the findings of NICE-SUGAR are widely generalizable.

However, the failure of IIT to improve outcomes when evaluated in a multi-centre study also raises an interesting question: Does this therapy truly contribute to patient harm, or is this more an issue of applicability-where less experienced centres are unable to reproduce the findings of Van den Berghe et al.? $?^{3,4}$

\section{Clinical perspective}

The NICE-SUGAR trial, a well-designed and well-conducted "effectiveness" trial ${ }^{1}$ in a large heterogeneous cohort of critically ill patients, found that IIT titrated to achieve a BG target between 4.5 and $6.0 \mathrm{mmol} \cdot \mathrm{L}^{-1}$ was associated with an increased risk of 90-day mortality compared with achieving a more conservative BG target of $<10.0 \mathrm{mmol} \cdot \mathrm{L}^{-1}$. This observation translates into a number needed to harm (NNH) of 39 (95\% CI, 21-263). For every 39 critically ill patients treated with IIT to achieve TGC, one additional patient would die by 90 days beyond what would have occurred when receiving conservative BG control. Importantly, no additional benefits of applying IIT were observed in this trial, including no observed differences in duration of mechanical ventilation, need for or duration of RRT, reduction in new organ dysfunction, positive blood cultures, rate of blood transfusion, ICU length of stay, or duration of hospitalization. Therapy with IIT was, however, associated with a marked increased risk of severe hypoglycemia (NNH 16, 95 CI, 14-19). For every 16 critically ill patients receiving IIT, one additional patient would suffer an episode of severe hypoglycemia beyond that expected from conservative BG control.

In conclusion, the findings of the NICE-SUGAR trial would suggest that the use of IIT to achieve a target BG $<6.0 \mathrm{mmol} \cdot \mathrm{L}^{-1}$ is associated with an increased risk of death and severe hypoglycemia. Moreover, NICE-SUGAR did not confirm the improved outcomes associated with IIT in the two single centre trials by Van den Berghe et al. These findings are certain to change clinical practice. Glycemic control will remain an important aspect in the overall management of critically ill patients when considering the adverse outcomes associated with hyperglycemia; however, the key message from the NICE-SUGAR trial 
would suggest a higher target of $\mathrm{BG}\left(<10.0 \mathrm{mmol} \cdot \mathrm{L}^{-1}\right)$ will contribute to similar outcomes while mitigating the potential for harm.

Financial disclosures None declared.

Conflicts of interest None declared.

\section{References}

1. Finfer $S$, Chittock $D R, S u S Y$, et al. Intensive versus conventional glucose control in critically ill patients. The NICE-SUGAR Study Investigators. N Engl J Med 2009; 360: 1283-97.

2. Finney SJ, Zekveld C, Elia A, Evans TW. Glucose control and mortality in critically ill patients. JAMA 2003; 290: 2041-7.

3. Van den Berghe $G$, Wilmer A, Hermans $G$, et al. Intensive insulin therapy in the medical ICU. N Engl J Med 2006; 354: 449-61.

4. Van den Berghe $G$, Wouters $P$, Weekers $F$, et al. Intensive insulin therapy in the critically ill patients. N Engl J Med 2001; 345: 1359-67.

5. Thomas G, Rojas MC, Epstein SK, Balk EM, Liangos O, Jaber $B L$. Insulin therapy and acute kidney injury in critically ill patients a systematic review. Nephrol Dial Transplant 2007; 22: 2849-55.

6. Hermans $G$, Wilmer A, Meersseman W, et al. Impact of intensive insulin therapy on neuromuscular complications and ventilator dependency in the medical intensive care unit. Am J Respir Crit Care Med 2007; 175: 480-9.

7. Dellinger RP, Carlet JM, Masur H, et al. Surviving Sepsis Campaign guidelines for management of severe sepsis and septic shock. Crit Care Med 2004; 32: 858-73.

8. Heyland DK, Dhaliwal R, Drover JW, Gramlich L, Dodek P, Canadian Critical Care Clinical Practice Guidelines Committee. Canadian clinical practice guidelines for nutrition support in mechanically ventilated, critically ill adult patients. JPEN J Parenter Enteral Nutr 2003; 27: 355-73.

9. Klaff LS, Wisse BE. Current controversy related to glucocorticoid and insulin therapy in the intensive care unit. Endocr Pract 2007; 13: 542-9.
10. Angus DC, Abraham E. Intensive insulin therapy in critical illness. Am J Respir Crit Care Med 2005; 172: 1358-9.

11. Vanhorebeek I, Langouche L, Van den Berghe G. Tight blood glucose control with insulin in the ICU: facts and controversies. Chest 2007; 132: 268-78.

12. Devos P, Preiser JC. Current controversies around tight glucose control in critically ill patients. Curr Opin Clin Nutr Metab Care 2007; 10: 206-9.

13. Wiener RS, Wiener DC, Larson RJ. Benefits and risks of tight glucose control in critically ill adults: a meta-analysis. JAMA 2008; 300: 933-44.

14. Brunkhorst FM, Engel $C$, Bloos $F$, et al. Intensive insulin therapy and pentastarch resuscitation in severe sepsis. N Engl J Med 2008; 358: 125-39.

15. Bilotta F, Caramia R, Cernak I, et al. Intensive insulin therapy after severe traumatic brain injury: a randomized clinical trial. Neurocrit Care 2008; 9: 159-66.

16. Bilotta F, Spinelli A, Giovannini F, Doronzio A, Delfini R, Rosa $G$. The effect of intensive insulin therapy on infection rate, vasospasm, neurologic outcome, and mortality in neurointensive care unit after intracranial aneurysm clipping in patients with acute subarachnoid hemorrhage: a randomized prospective pilot trial. J Neurosurg Anesthesiol 2007; 19: 156-60.

17. Oksanen T, Skrifvars MB, Varpula T, et al. Strict versus moderate glucose control after resuscitation from ventricular fibrillation. Intensive Care Med 2007; 33: 2093-100.

18. Preiser JC, Devos $P$. Clinical experience with tight glucose control by intensive insulin therapy. Crit Care Med 2007; 35: S503-7.

19. Bellomo R, Egi M. Glycemic control in the intensive care unit: why we should wait for NICE-SUGAR. Mayo Clin Proc 2005; 80: $1546-8$.

20. Arabi YM, Dabbagh OC, Tamim HM, et al. Intensive versus conventional insulin therapy: a randomized controlled trial in medical and surgical critically ill patients. Crit Care Med 2008; 36: 3190-7.

21. Van den Berghe $G$, Bouillon $R$, Mesotten D. Glucose control in critically ill patients. N Engl J Med 2009; 361: 89. 